\title{
Discless Accretion in Intermediate Polars?
}

\author{
David A.H. Buckley \\ South African Astronomical Observatory, PO Box 9, Observatory 7935, \\ Cape Town, South Africa
}

\begin{abstract}
I review the evidence for disc and discless accretion in the asynchronous magnetic CVs, the intermediate polars (IPs). The presence and relative amplitudes of the spin and synodic (beat) X-ray periods, and related orbital sidebands, are very model dependent, and support disc accretion dominating in all the well-studied IPs. A component of non-disc accretion is sometimes observed, a result of stream overflow penetrating to the magnetosphere, and is responsible for the synodic period. The recently discovered polarized IP, RX J1712.6-2414, is a prime candidate (the first) for purely discless, stream-fed accretion in such a system. While the polarisation varies at the spin period, the $\mathrm{X}$-ray variations occur at the synodic period, a result of the modulation in the mass transfer rate for a discless accretor.
\end{abstract}

\section{Introduction}

Intermediate polars (IPs) are magnetic CVs $(\mathrm{mCVs})$ emitting $\mathrm{X}$-rays $\left(L_{X} / L_{\text {opt }}\right.$ 1) as a result of accretion (see Patterson 1994, Warner 1995 for recent reviews). As in all $\mathrm{mCVs}$, magnetically channelled gas forms a strong shock in the proximity of the magnetic poles of a white dwarf. The gas is heated to high temperatures, and cools predominantly via thermal bremsstrahlung and cyclotron emission.

Unlike the other members of the magnetic subclass, the polars (also called AM Herculis stars), the white dwarf's rotation is not synchronized with the orbital period. Speculation that IPs, which as a group have orbital periods longer than polars, eventually become polars, after loss of sufficient orbital angular momentum (e.g. Chanmugam \& Ray 1984), has to a large extent been contradicted observationally. Central to all the arguments concerning the evolution of $\mathrm{mCVs}$ is the strength of the magnetic fields in IPs. If IPs evolve into polars, then they must have similar magnetic field strengths to polars.

The strong magnetic fields ( $8-70 \mathrm{MG}$ ) in polars (Cropper 1990) is evidenced by circularly polarized optical and IR emission (up to 60\%), cyclotron lines and Zeeman features. In comparison only three of the 15 or so IPs have a measurable circular polarization, and cyclotron or Zeeman features have never been seen. Estimates of IP magnetic fields have until recently relied on indirect methods based on either the rate of change of the spin period, or the assumption of spin equilibrium (e.g. Patterson 1994). Currently evidence of this nature points to lower magnetic field strengths in the IPs. 


\section{Implications from the presence of a disc}

A crucial question regarding magnetic field strengths of IPs is whether accretion discs are present. The formation of a disc in a $\mathrm{mCV}$ is prevented when the distance at which the accretion stream from the secondary star couples to the white dwarf's magnetic field exceeds a critical value. The coupling distance is referred to as the magnetospheric radius for stream accretion, $r_{\mu, s t r}$, while the critical distance is usually taken to be the circularization radius, $r_{r}$ (e.g. Warner 1995). The latter is simply the radius at which material orbiting the white dwarf has the same angular momentum as it did when it left the inner Lagrangian point $\left(L_{1}\right)$.

The condition for discless accretion can be formulated as an expression for the limit on the magnetic field of the primary:

$$
B_{6}(\mathrm{MG}) \gtrsim 10.7 \eta P_{\text {orb }}^{1.17} \dot{M}_{17}^{0.5} M_{1}^{3.26},
$$

which is derived from the expression for the magnetic moment (Wickramasinghe, Wu \& Ferrario 1991; Warner 1995). The parameter $\eta\left(=r_{r} / r_{L 1}\right)$ is a function of the mass ratio, and is $\lesssim 0.4$ (Warner 1995). An appropriate white dwarf mass-radius relation, $R_{9}(\mathrm{~cm})=0.55 M_{1}^{-0.81}$ for Hamada-Salpeter Carbon white dwarfs (Buckley \& Shafter 1995), was used to eliminate the white dwarf radius from the magnetic moment expression. The expression can be used to put an upper limit on the magnetic field for systems in which it can be demonstrated that an accretion disc is present.

\section{Direct evidence for discs in IPs}

The evidence for accretion discs in IPs includes observations of eclipses, rotational disturbances and disc bright-spot s-waves, and is reviewed by Hellier (1991). Whereas only one IP has both X-ray and optical eclipses (EX Hya), three others (TV Col, FO Aqr \& AO Psc) have shallow partial optical eclipses. These are easily explained by the presence of an accretion disc. Emission line "rotational disturbances", arising from partial eclipses of the line emitting region in a disc, are seen in both EX Hya and FO Aqr. Perhaps the strongest evidence is the detection of "s-waves" in most IP spectra, attributed to line emission from a bright-spot on a disc, where the accretion stream impacts it, just as in a non-magnetic disc system.

\section{Accretion stream-disc overflow}

Certain observed features in IPs can be explained if part of the accretion stream overflows the disc. Lubow (1989) investigated stream overflow, and concluded that part of the stream could continue to follow its free-fall trajectory, and impact the disc at some point close to the white dwarf. In at least three IPs there is strong evidence for disc-overflow accretion: EX Hya and V795 Her both exhibit a higher amplitude emission line s-wave (Hellier et al. 1989; Haswell et al. 1993), while FO Aqr shows an absorption component (Hellier, Mason \& Cropper 1990). In both cases the region responsible for the emission or absorption coincides with 
a down-stream part of the accretion stream which has overflowed the disc. In non-magnetic systems, the stream re-impacts the disc close to the white dwarf, but in IPs the stream is more likely to encounter the magnetosphere boundary. This provides direct accretion into the magnetosphere, resulting in a modulation at the synodic period of the accretion onto the magnetic poles.

\section{X-ray power spectra: accretion discriminants}

If the magnetic fields in IPs are sufficiently strong to prevent an accretion disc from forming, then direct accretion (i.e. not mediated by a disc) from the mass transferring stream occurs. The stream will follow its free-fall trajectory until it reaches the magnetosphere, where it becomes coupled to the magnetic field lines. The major observational consequence is that the X-ray emission should be modulated predominantly at the synodic frequency, $\omega-\Omega$ (i.e. the beat frequency between the white dwarf spin $(\omega)$ and orbital $(\Omega)$ frequencies). Wynn \& King (1992) discussed the appearance of X-ray power spectra for discless accretion models, and concluded that periods corresponding to the $\omega-\Omega$ and $2 \omega-\Omega$ sideband frequencies should usually dominate, except in certain circumstances (e.g. for off-set dipole geometries). Recent simulations by Taylor, Norton \& Beardmore (1995) show that the synodic period will dominate for discless accretion, and even for a "hybrid" model consisting of both disc and discless accretion, while the spin period will always dominate for pure disc accretion.

What do the X-ray observations of IPs reveal ? In all but one case, the Xrays are modulated predominantly at the spin frequency, $\omega$, and the sidebands are either weak, transient or not present (e.g. Hellier 1992). The exception, TX $\mathrm{Col}$, has shown a hard X-ray synodic periodicity dominating over the spin modulation (Buckley \& Tuohy 1989). This object was suggested to be the first case of direct discless accretion (Mason et al. 1988), although other evidence suggests the presence of an accretion disc (Buckley \& Tuohy 1989). Most IPs studied in sufficient detail (e.g. FO Aqr, V1223 Sgr, AO Psc, BG CMi) have, on at least one occasion, shown a synodic variation or related sideband. This behaviour maybe evidence that these IPs are examples of hybrid systems, involving both accretion modes. The changes are likely a result of variations in the accretion geometry, with the direct stream-fed accretion becoming less important. The dominance of the spin period in the X-ray power spectra of IPs implies disc accretion predominates, although not exclusively.

\section{RX J1712.6-2414: a discless accretor?}

A recently discovered (Buckley et al. 1995) polarized IP, RX J1712.6-2414, has a strong synodic X-ray period, dominating the spin, and is therefore the best candidate so far amongst IPs for a discless accretor (Buckley et al. 1996). RX $\mathrm{J} 1712.6-2414$ is unusual in exhibiting strong circular polarization (up to $-5.1 \%$ ) which is pulsed at the 927 -s spin period, with a semi-amplitude increasing with wavelength to $\sim 1 \%$ in the red $(7000 \AA)$. Only one other IP, the unusually soft Xray system PQ Gem, shows pulsed polarization (Piirola, Hakala \& Coyne 1993). In their discovery paper, Buckley et al. (1995) attempted to model the polarized flux, and determined a magnetic field strength of $\sim 8 \mathrm{MG}$. Further modelling by 
Väth (1996) indicates an even stronger field. Using equation (1) with $P_{\text {orb }}=$ $3.41 \mathrm{~h}, \eta<0.21(0.4<q<1), \dot{M}_{17}=1$ and $M_{1}=1$ (typical values for an IP), gives a magnetic field strength of $\sim 8$ MG. Buckley et al. $(1995,1996)$ concluded that the magnetic field derived from the polarization measurement was sufficient to prevent disc formation.

However, the most conclusive evidence comes from ROSAT observations (Buckley et al. 1996), which show that the X-rays are modulated strongly at the 1003-s synodic period with a semi-amplitude of $15 \%$, compared to $\lesssim 4 \%$ for any spin modulation. RX J1712.6-2414 is therefore the first case of an IP which fulfills the predictions of discless accretion. The synodic period is also occasionally seen in the optical photometry and radial velocities, whereas the spin period is only detected in the circular polarimetry (Buckley et al. 1995). Direct accretion into the magnetosphere of a low inclination IP, at a modest dipole tilt angle $\left(\leqslant 10^{\circ}\right)$, is the best model for RX J1712.6-2414, and is capable of explaining the variety of observational features. Model simulations also support this conclusion, though the limits placed on the aspect geometry are rather strict (Garlick 1996). It is still possible that a minor component of the accretion proceeds via a disc in RX J1712.6-2414, and this will be investigated by future X-ray observations with $R X T E$.

\section{References}

Buckley, D.A.H. \& Tuohy I.R. 1989, ApJ, 344, 376

Buckley, D.A.H. \& Shafter, A.W. 1995, MNRAS, 275, L61

Buckley, D.A.H., et al. 1995, MNRAS, 275, 1028

Buckley, D.A.H., et al. 1996, MNRAS, submitted

Cropper, M.S. 1990, Space Sci. Rev., 54, 195

Chanmugam, G. \& Ray, A. 1984, ApJ, 285, 252

Garlick, M.A. 1996, MNRAS, in press

Haswell, C. et al. 1994, ASP Conf. Ser. 56, 268

Hellier, C. 1991 MNRAS, 251, 693

Hellier, C. 1992, MNRAS, 258, 578

Hellier, C. et al. 1989, MNRAS, 238, 1107

Hellier, C., Mason, K.O. \& Cropper, M.S. 1990, MNRAS, 242, 250

Lubow, S. 1989, ApJ, 340, 1064

Mason, K.O., Rosen, S.R. \& Hellier, C. 1988, Adv. Space Res., 8(2), 293

Patterson, J. 1994, PASP, 106, 209

Piirola, V., Hakala, P. \& Coyne, G.V. 1993, ApJ, 410, L107

Taylor, P., Norton, A.J. \& Beardmore, A.P. 1995, ASP Conf. Ser., 85, 202

Väth, H. 1996, A\&A, in press

Warner, B. 1995, Cataclysmic Variable Stars. Cambridge University Press, Cambridge, p. 376

Wickramasinghe, D.T., Wu, K. \& Ferrario, L. 1991, MNRAS, 249, 460

Wynn, G. \& King, A.R. 1993, MNRAS, 255, 83 